\title{
Interfaz de Simulación de la Cinemática de Robots Manipuladores Seriales
}

\author{
Washington Caraguay Ambuludi / Luis Benavides Castillo
}

\section{Resumen}

En este trabajo se desarrolla una interfaz gráfica de simulación de robots manipuladores capaz de simular la cinemática directa y diferencial de robots seriales con un rango entre dos y seis grados de libertad (GdL); dicha interfaz ha sido desarrollada en Matlab con base en los modelos matemáticos de su movimiento. Con ese objeto, primero se expone el desarrollo de la robótica industrial y sus fundamentos teóricos. Luego se detallan las ecuaciones matemáticas que modelan su cinemática para desarrollar posteriormente un programa de simulación. Finalmente, con base en los resultados se realiza un análisis cinemático del robot IRB 2400 de la firma ABB. Este artículo pretende contribuir con la caracterización del comportamiento en las etapas previas y posteriores al diseño y construcción de robots manipuladores seriales industriales, prescindiendo físicamente del robot.

\section{Palabras clave:}

Simulación, robots, manipuladores, cinemática, grados de libertad.

\begin{abstract}
This paper presents a graphical interface simulation for robot manipulators able to simulate direct, inverse and differential kinematics of serial robots with a range between two and six degrees of freedom (DoF). This was developed in Matlab based on mathematical models of motion. For this purpose, the theoretical bases and industrial robotics development are expounded with mathematics equations that model their kinematics model and a simulation program was developed. Finally, an IRB 2400 ABB simulated robot analysis is achieved. This article aims to contribute to the characterization of the design behavior and construction of industrial serial manipulators robot regardless of the robot's physical form.
\end{abstract}

Keywords:

Simulation, robots, manipulators, kinematic, degrees of freedom. 


\section{Introducción}

Un robot manipulador serial industrial también conocido como brazo manipulador, es una estructura mecánica consistente en una secuencia de eslabones conectados por medio de articulaciones, que se caracteriza por un brazo que asegura movilidad, una muñeca que confiere destreza y un efector final que se mueve dentro de un espacio de trabajo y se encarga de realizar las tareas requeridas por el robot (Bravo \& Reyes, 2009). La simulación es una herramienta utilizada para evaluar, predecir y reproducir el comportamiento de los robots en su movimiento, además de verificar y optimizar la planificación de la trayectoria del proceso. La simulación utilizando un computador mediante un software apropiado es un complemento en el desarrollo de procesos, previo a la etapa experimental.

Para el desarrollo de una simulación es necesario conocer el modelo exacto del sistema; por lo que se requiere un lenguaje de programación matemático de alto nivel como lo es el software MATLAB, el mismo que posee un entorno gráfico amigable al usuario a través de herramientas y utilerías para el desarrollo de sistemas aplicados a la robótica.

En este sentido, la necesidad de analizar características físicas de los robots manipuladores seriales industriales, conlleva a la realización de este artículo, mismo que trata en detalle del desarrollo de una interfaz gráfica para la simulación de los modelos cinemáticos de robots manipuladores, desde dos hasta seis grados de libertad. Para su implementación se ha hecho uso de las ecuaciones matemáticas que modelan la cinemática inversa, directa y diferencial. Es decir, en el espacio de trabajo, la cinemática rotacional de un robot manipulador sufre transformaciones numéricas para llevar a cabo la ejecución de una determinada tarea; lo que conduce a la representación matemática de posiciones y orientaciones de las piezas, las herramientas y del mecanismo en sí, para lo cual se debe definir sistemas de coordenadas y desarrollar convenciones para su manipulación.

Más aún, con el objeto de validar la herramienta, se utiliza la geometría del robot IRB 2400 del fabricante ABB para la simulación, se muestran resultados y se analiza su comportamiento cinemático directo y diferencial. Así mismo, la interfaz muestra su facilidad de uso y versatilidad para su aplicación. Finalmente se exponen algunas conclusiones respecto al proceso de simulación.

\section{Desarrollo de la Robótica Industrial}

El actual desarrollo de robots manipuladores industriales no se produjo sino hasta después de 1940. La aparición de este tipo de robots se debió inicialmente a la necesidad de manipular materiales peligrosos y en la exploración del espacio y más tarde para lograr flexibilizar la automatización. Durante la década de 1940, la Oak Ridge y Argonne National Laboratories empezaron el desarrollo de manipuladores maestro-esclavo controlados de forma remota para la manipulación de materiales radioactivos (Lung-Wen, 1999). Estos sistemas de tipo maestro-esclavo, fueron diseñados para reproducir fielmente los movimientos de mano y brazo realizados por un operario humano. El trabajo sobre estos manipuladores fue seguido rápidamente por sistemas más sofisticados capaces de realizar operaciones repetitivas autónomas.

A mediados de los años cincuenta, George C. Devol desarrolló un dispositivo que él llamó dispositivo de transferencia programada y articulada, un manipulador cuya operación podía ser programada y que podía seguir una secuencia de pasos de movimientos determinados por las instrucciones en el programa. Posteriores desarrollos de este concepto por Devol y Joseph F. Engelberger condujo al primer robot industrial, introducido por Unimation Inc. en 1959 (Fu, González \& Lee, 1988).

En 1969 en la Universidad de Stanford, 
el estudiante de Ingeniería Mecánica Víctor Scheinman desarrolla el Robot Stanford, un brazo robótico controlado por computador. Cuatro años más tarde, en Cincinnati Milacron Corporation, Richard Hohn desarrolló el robot llamado The Tomorrow Tool, or T3. El T3 fue el primer robot industrial disponible comercialmente controlado por un microordenador, así como el primer robot americano para utilizar esta revolucionada configuración. Accionado hidráulicamente, el T3 se utiliza en aplicaciones tales como la soldadura de carrocerías de automóviles, parachoques de automóviles, la transferencia y la carga de máquinas y herramientas. En 1975, el T3 se introdujo para aplicaciones de perforación y en el mismo año, se convirtió en el primer robot para ser utilizado en la industria aeroespacial.

En 1970 en Europa, el Grupo sueco ASEA hizo importantes avances en robots eléctricos. Para hacer frente a operaciones de rectificado automático, ASEA introdujo los robots IRB 6 e IRB 60 totalmente eléctricos en 1973. Dos años más tarde, ASEA fue el primero en instalar un robot en una fundidora de hierro. Para 1977 ASEA introdujo dos robots industriales más con motor eléctrico, ambos de los cuales utilizan microcomputadoras para su programación y funcionamiento. En 1988, ASEA se fusionó con la BBC, Brown Boveri. Ltd. de Baden, Suiza, para formar ABB (ASEA Brown Boveri), actualmente uno de los líderes mundiales en energía y tecnología de automatización (Kurfess, 2005).

En 1979 en Japón, en la Universidad de Yamanashi, IBM y Sankyo unieron sus fuerzas y conocimientos para desarrollar el Selective Compliance Assembly Robot Arm, SCARA, mismo que fue diseñado con articulaciones de giro que tenían ejes verticales, proporcionando así rigidez en la dirección vertical. La pinza se controló utilizando control de fuerza, mientras que el resto de articulaciones eran operados utilizando control de posición. Sobre esta base geométrica de SCARA, Adept Technology fue fundada en 1983, año en que lanza el Adept One Direct Drive Robot, el mismo que consiste en un robot de 4 grados de libertad, con dos motores localizados en la base que producían el movimiento del eslabón horizontal y dos motores localizados en el antebrazo y el extremo que hacen de desplazamiento y rotación de la pinza de agarre. Dado que el movimiento de sus eslabones está restringido dentro de un plano horizontal, no actúa ninguna carga de gravedad sobre sus dos actuadores. Estos robots son ampliamente utilizados para las operaciones de montaje, particularmente para tareas planas (Asada \& Youcef-Tomi, 1987).

En la actualidad, los robots manipuladores SCARA, además de ser utilizados en la experimentación, son muy rápidos e ideales para operaciones en las que los requisitos de movimiento vertical, son pequeños en comparación con los requisitos de movimiento horizontal, como es el trabajo de montaje o desmontaje conocido como pick \& place (Caraguay, 2013). Sobre este modelo de robot se han realizado muchas pruebas sobre algoritmos de control, con la finalidad de mejorar el comportamiento dinámico del manipulador, como los que se han realizado en (Ertugrul \& Kaynak, 1997; Vivas, 2006; Muñoz, Mosquera \& Gaviria, 2011).

Spong, Hutchinson \& Vidyasagar (2006) proporcionan la siguiente definición oficial de robot según el Instituto Americano de Robótica (RIA): "Un robot es un manipulador multifuncional reprogramable, diseñado para mover, materiales, piezas, herramientas o dispositivos especializados, a través de movimientos variables, programados para la realización de diversas tareas" (pág. 2). De manera similar, Kelly, Santibáñez \& Loría, (2005) exponen la definición adoptada por la Federación Internacional de Robótica bajo la norma ISO/TR 8373: "Un robot manipulador industrial es una máquina manipuladora con varios 
grados de libertad controlada automáticamente, reprogramable y de múltiples usos, pudiendo estar en un lugar fijo o móvil para su empleo en aplicaciones industriales" (p.4). Dada las dos definiciones anteriores de robots manipuladores se puede inferir que coinciden en que los robots son máquinas controlables, programables y de múltiples usos.

Morfológicamente, los robots manipuladores seriales se componen de varias articulaciones unidas entre sí, poseen una dinámica altamente no lineal con un fuerte acoplamiento entre sus respectivas articulaciones, que conlleva un alto grado de dificultad en su control. Muchas estrategias son utilizadas para el control de brazos robóticos en los últimos años, como se indica en (Canudas, Siciliano \& Bastin, 1996; Lewis, Abdallah \& Dawson, 1993; Samson, Le Borgne \& Espinau, 1991).

Los robots manipuladores, se emplean para trabajar con artefactos externos a su arquitectura. A las partes móviles o articulaciones mecánicas se les llama grados de libertad. Un efector final, o actuador final es el elemento que se coloca en el último enlace del robot manipulador y que suministra la capacidad de agarre del objeto que se pretende manipular, o la colocación de una herramienta apropiada para la tareas: como pintura, soldadura, paletización, pick \& place, entre otras. Aunque comercialmente existen algunas configuraciones como paralelas, cartesianas, SCARA, cilíndricos, esféricos; la gran mayoría de robots industriales poseen configuración antropomórfica, presentando una mayor destreza en su espacio de trabajo debido a que sus eslabones están unidos por articulaciones rotacionales (Siciliano, Sciavicco, Villani \& Oriolo, 2009).

Es de considerar que los robots manipuladores son sistemas mecánicos que involucran una dinámica, misma que debe ser modelada para explicar todos los fenómenos físicos que se encuentran en su estructura mecánica. Un modelo dinámico completo del robot debe incluir no sólo la inercia de los propios eslabones del mecanismo; sino también la de los actuadores y sistemas de transmisión de movimiento (Caraguay, 2013).

Si se analiza la construcción y el avance tecnológico, se pueden clasificar a los robots como tradicionales y de transmisión directa. Los robots tradicionales emplean engranes y reductores para incrementar la capacidad limitada del par en sus motores y reducir la velocidad rotacional de los mismos. Sin embargo, la desventaja radica en que el sistema de engranes produce fenómenos de elasticidad y fricción en las articulaciones. Esta situación representa el principal inconveniente de los sistemas de engranajes, debido a que convierten la energía mecánica en energía térmica, degradando las partes mecánicas de los engranes, lo cual repercute en errores de posicionamiento y bajo rendimiento del robot. Los pares de fricción pueden ser de considerable magnitud como para predominar sobre la dinámica del manipulador.

Por su parte la tecnología de los robots de la transmisión directa se basa en que el servomotor funciona como una fuente ideal de par, no hay pérdidas de energía como sucede en los robots tradicionales, simplificando el sistema de engranaje tradicional, de ahí que el motor está directamente acoplado al eslabón, lo cual elimina el cascabeleo, o falta de movimiento y reduce significativamente el fenómeno de la fricción; ya que el motor no tiene escobillas. Además, la construcción mecánica del robot es más simple y la exactitud en el posicionamiento final es mejorada.

Una característica importante de la tecnología de transmisión directa es la electrónica asociada al motor que lo hace funcionar como fuente ideal de par aplicado, lo cual significa que independientemente de la carga mecánica mantiene constante el par 
solicitado en cada periodo de muestreo. Sin embargo, en la práctica está restringido por los límites físicos en los servos actuadores (Reyes, 2011).

\section{La simulación como herramienta de pruebas}

La simulación utilizando un computador mediante un software apropiado, es un complemento en el desarrollo de procesos experimentales de sistemas robotizados. Para el desarrollo de una simulación es necesario conocer el modelo matemático exacto del sistema. La importancia de la simulación consiste en mostrar comportamientos del sistema antes de llevarlo a la práctica, algo que no se podría lograr evidenciando ecuaciones o datos numéricos. Reyes (2011) afirma que "las simulaciones son importantes durante las primeras etapas del diseño de algoritmos de control” (pág. 316), exceptuando aspectos prácticos mecánicos.

Una de las contribuciones más relevantes consiste en que un simulador brinda seguridad protegiendo la integridad del usuario y de su entorno. Muchas simulaciones han sido desarrolladas con base en el modelo matemático del sistema, como ejemplo de simulaciones realizadas de sistemas robotizados tenemos a (Morales \& Graciano Cruz, 2002; Bravo \& Reyes, 2009; Hoyo, Reyes, Rebolledo \& Espinoza, 2009, Martínez \& Salazar, 2007) con la finalidad de lograr una herramienta que permita diseñar y probar robots manipuladores de diversos tipos, así como también de material didáctico y de investigación en los cursos académicos de asignaturas de ciencias e ingeniería. Por tanto, uno de los propósitos de simular es comprender el comportamiento de un sistema previo a sus etapas de operación, sin recurrir al uso del sistema físico real con base en algoritmos y relaciones matemáticas.

\section{E1 Robot IRB 2400}

El IRB 2400 es el robot industrial más popular del mundo, Figura 1. Su estructura robusta y mínimo uso de piezas, contribuyen a su gran fiabilidad y largos intervalos entre periodos de mantenimiento. En sus diferentes versiones y con la mejor exactitud, proporciona un excelente rendimiento en aplicaciones de manipulación de materiales, alimentación de máquinas y aplicaciones de proceso.

El IRB 2400 le ofrece un mayor índice de producción, tiempos de puesta en producción reducidos y una entrega más rápida de sus productos manufacturados. El IRB 2400 posee configuración antropomórfica y entre las aplicaciones típicas figuran: asistencia de maquinaria, manipulación de material, soldadura por arco y otras aplicaciones de procesos. Actualmente, ABB ha lanzado la gama de robots de cuarta generación, con el IRB 2600 a la cabeza cuyas características de menor peso, capacidades de carga útil entre 12 y $20 \mathrm{~kg}$, mayores aceleraciones y un avanzado control de movimiento, han relegado al IRB 2400. Sin embargo, IRB 2400 por sus niveles de productividad en la industria, aún sigue siendo ampliamente utilizado (ABB Robotics, 2015).

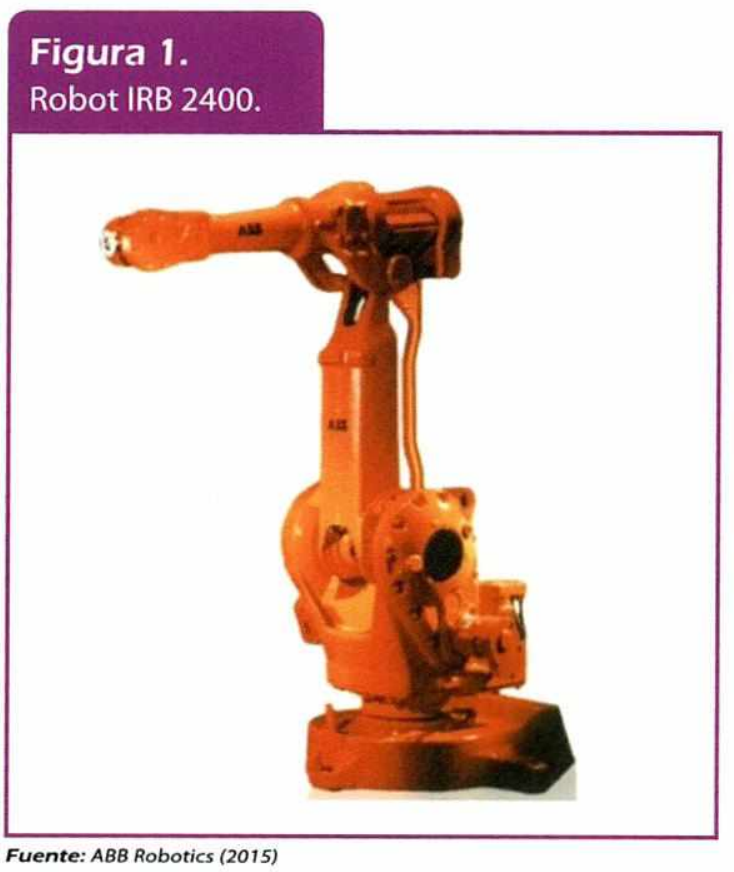

Además, la página web del fabricante proporciona información de la geometría 
del robot así, como sus límites de rango de trabajo, Figura 2, información que se requiere para las pruebas de simulación.

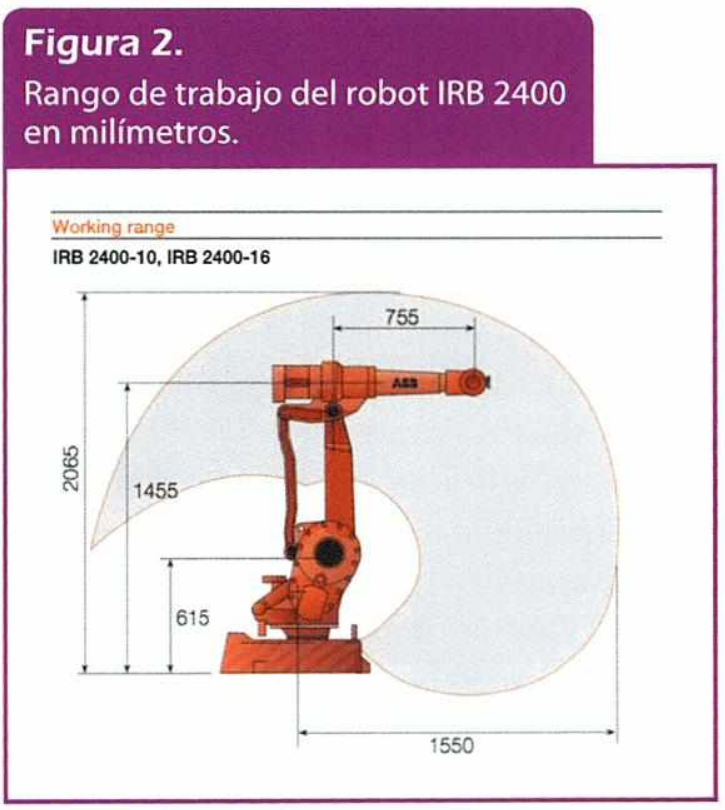

Fuente: ABB Robotics (2015)

\section{Modelo Cinemático Directo y Dife- rencial del Robot IRB 2400}

La resolución del problema cinemático directo, permite conocer cuál es la posición y orientación que adopta el extremo del robot cuando cada una de las variables que fijan la posición u orientación de sus articulaciones toma valores determinados. Haciendo uso de las características geométricas presentadas en la Figura 2, y del método Denavith-Hartenberg (Denavit \& Hartenberg, 1955), se obtendrá la matriz " $T$ " de transformación homogénea mostrada en las ecuaciones (1), (2) y (3), que permiten resolver completamente el problema de cinemática directa (Denavit \& Hartenberg, 1955; Spong \& Vidyasagar, 1989; Lung-Wen, 1999; Barrientos, Peñín, Balaguer, \& Aracil, 2007)

$$
\begin{gathered}
T={ }_{1}^{0} A{ }_{2}^{1} A{ }_{3}^{2} A{ }_{4}^{3} A{ }_{5}^{4} A{ }_{6}^{5} A . \\
T={ }_{6}^{0} A . \\
{ }_{i}^{i-1} A=\left[\begin{array}{cccc}
\cos \theta_{\mathrm{i}} & -\cos \alpha_{\mathrm{i}} \sin \theta_{\mathrm{i}} & \sin \alpha_{\mathrm{i}} \sin \theta_{\mathrm{i}} & \mathrm{a}_{\mathrm{i}} \cos \theta_{\mathrm{i}} \\
\sin \theta_{\mathrm{i}} & \cos \alpha_{\mathrm{i}} \cos \theta_{\mathrm{i}} & -\sin \alpha_{\mathrm{i}} \cos \theta_{\mathrm{i}} & \mathrm{a}_{\mathrm{i}} \sin \theta_{\mathrm{i}} \\
0 & \sin \alpha_{\mathrm{i}} & \cos \alpha_{\mathrm{i}} & d_{\mathrm{i}} \\
0 & 0 & 0 & 1
\end{array}\right] . \\
T={ }_{1}^{0} A{ }_{2}^{1} A{ }_{3}^{2} A{ }_{4}^{3} A{ }_{5}^{4} A{ }_{6}^{5} A . \\
T={ }_{6}^{0} A .
\end{gathered}
$$

El primer paso para el modelado cinemático, es la asignación adecuada de coordinar marcos de referencia coordenados a cada eslabón, donde cada sistema es ortogonal y los ejes obedecen a la regla de la mano derecha (Kin-Huat, 2007).

Considerando la geometría expuesta en la Figura 2, las medidas $L_{1}, L_{2}, L_{3}$ y $L_{4}$ son presentadas en el apartado numérico (4).

$L_{1}=615 \mathrm{~mm}=0.615 \mathrm{~m}$

$L_{2}=840 \mathrm{~mm}=0.840 \mathrm{~m}$

$L_{3}=755 \mathrm{~mm}=0.755 \mathrm{~m}$

$L_{4}=100 \mathrm{~mm}=0.100 \mathrm{~m}$

(Medida aproximada del efector final)

Estos valores servirán de referencia para lograr la simulación ya que corresponden a las características geométricas del Robot IRB 2400. Con estas consideraciones, para el desarrollo del modelo se empieza con un esquema referencial del robot, relacionando un sistema de referencia para cada una de sus articulaciones, que son mostradas en la Figura 3. Por otro lado, al aplicar la representación Denavith-Hartenberg, se obtuvieron los parámetros ( $\theta \mathrm{i}, \mathrm{di}, \mathrm{ai}, \mathrm{ai})$, mismos que se muestran en la Tabla 1.

\section{Figura 3. \\ Esquema referencial del Robot IRB 2400.}

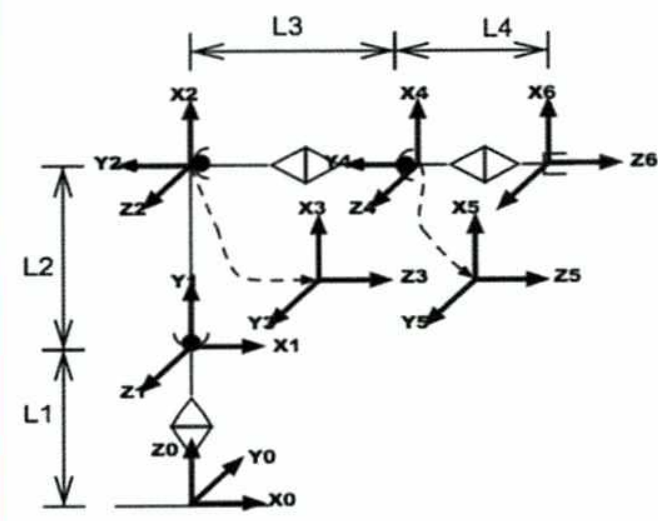

Fuente: Elaboración propia. 


\section{Tabla \#1}

\begin{tabular}{|l|l|l|l|r|}
\hline \multicolumn{5}{|c|}{$\begin{array}{l}\text { Parámetros obtenidos al aplicar el método } \\
\text { Denavith-Hartemberg }\end{array}$} \\
\hline MTH & $\theta_{i}$ & $d_{i}$ & $a_{i}$ & $a_{i}$ \\
\hline${ }_{1}^{0} \mathrm{~A}$ & $q_{1}$ & $L_{1}$ & 0 & 90 \\
\hline${ }_{2}^{1} \mathrm{~A}$ & $q_{2}+90$ & 0 & $L_{2}$ & 0 \\
\hline${ }_{3}^{2} \mathrm{~A}$ & $q_{3}$ & 0 & 0 & 90 \\
\hline${ }_{4}^{3} \mathrm{~A}$ & $q_{4}$ & $L_{3}$ & 0 & -90 \\
\hline${ }_{5}^{4} \mathrm{~A}$ & $q_{5}$ & 0 & 0 & 90 \\
\hline${ }_{6}^{5} \mathrm{~A}$ & $q_{6}$ & $L_{4}$ & 0 & 0 \\
\hline
\end{tabular}

Nota: Los cuatro parámetros de Denavith Hartemberg $\left(\theta_{i}, d_{i}, a_{i}, \alpha_{i}\right)$ dependen únicamente de las características geométricas de cada eslabón y de las articulaciones que le unen con el anterior y siguiente: Rotación alrededor del eje $z_{i-1}$ un ángulo $\theta_{i}$. Traslación a lo largo de $z_{i-1}$ una distancia $d_{i}$. Traslación a lo largo de $x_{i}$ una distancia $a_{i}$. Rotación alrededor del eje $x_{i}$ un ángulo $\alpha_{i},($ Paul, 1981).

A partir de la información contenida en las matrices de transformación homogénea, que definen el modelo cinemático directo, se obtiene el modelo cinemático diferencial, que relaciona el espacio de velocidades del movimiento de las articulaciones, con el espacio de velocidades lineales y angulares del extremo del robot (Craig, 2006; Barrientos et al., 2007; Ollero, 2007; Hegde, 2008), este modelo está representado por su matriz Jacobiana, J; para su desarrollo se ha utilizado el método de propagación de velocidades, muy común en (Yoshikawa, 1990; Lung-Wen, 1999; Reyes, 2011), el mismo que permite obtener las columnas de la matriz Jacobiana a partir de las matrices ${ }_{i}^{i-1} A$ que define el modelo cinemático.

Para la interpretación de las ecuaciones(5), (6), (7) y (8), se denomina ${ }_{i}^{0} \mathrm{Z}$ al vector unitario orientado según el eje de la articulación $i+1$, definido en el sistema de coordenadas de la base del robot $\left(S_{0}\right)$. Además, las matrices ${ }_{i}^{i-1} A$ contienen la información de los vectores directores y origen del sistema $\left(S_{i}\right)$ en la base $\left(S_{i-1}\right)$. Por tanto, la matriz ${ }_{i}^{0} A$ contendrá la información de los vectores directores y origen del sistema $\left(S_{i}\right)$ en la base $\left(S_{0}\right)$. De modo que ${ }_{i}^{0} z$ estará definido por los tres primeros elementos de la tercera columna de ${ }_{i}^{0} A$. Como se evidencia en la ecuación (5):

$$
{ }_{i}^{0} Z={ }_{i}^{0} A(1: 3,3) \text {. }
$$

Así también se denominará ${ }_{n}^{i} p,(n=$ número de grados de libertad), al vector que va desde el origen del sistema $\left(S_{i}\right)$ hasta el extremo del robot expresado en el sistema de la base del robot $\left(S_{i}\right)$. Puesto que la cuarta columna de ${ }_{n}^{0} A$ contiene las coordenadas del extremo del robot en el sistema $\left(S_{0}\right)$ y la cuarta columna del ${ }_{i}^{0} A$ contiene las coordenadas del origen del sistema $\left(S_{i}\right)$ en el sistema $\left(S_{0}\right),{ }_{n}^{i} p$ se obtendrá restando las cuartas columnas de ${ }_{n}^{0} A$ y ${ }_{i}^{0} A$. Es decir se tiene la ecuación (6):

$$
{ }_{n}^{i} p={ }_{n}^{0} A(1: 3,4)-{ }_{i}^{0} A(1: 3,4) .
$$

Definidos ahora los vectores ${ }_{i}^{0} z$ y ${ }_{n}^{i} p$, la matriz Jacobiana geométrica, se puede obtener como una matriz de $6 \mathrm{x} \mathrm{n}$, expresada por columnas, en este caso al tratarse de un manipulador de 6 grados de libertad tenemos la ecuación (7):

$$
J=\left[J_{1} J_{2} \mid J_{3} J_{4} J_{5} J_{6}\right] .
$$

Sin embargo, hay que establecer si el eslabón es de rotación o traslación, y proceder como lo expresa la ecuación (8):

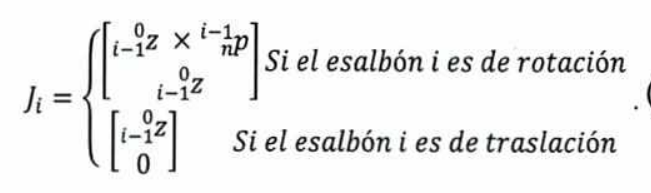

Una interpretación física de la Jacobiana, establece que cada columna de $J$ representa la dirección del vector velocidad, tanto lineal como angular del extremo originado por el giro de su correspondiente articulación. 
En consecuencia, si J tiene más filas que columnas, significa que el número de ejes del robot es inferior a la dimensión del espacio de la tarea y por lo tanto el movimiento del robot está sometido a ciertas restricciones, en otras palabras no se puede alcanzar cualquier orientación.

Por otro lado, las configuraciones singulares son aquellas en las que el determinante de J se anula. De esta manera, un incremento infinitesimal de las coordenadas cartesianas supondría un incremento infinito de las coordenadas articulares, lo que significa que el pretender que el extremo del robot se mueva a velocidad constante, obligaría mover las articulaciones a velocidades inabordables por sus actuadores. Es conocido que todos los manipuladores tienen singularidades en los límites, o fronteras de sus espacios de trabajo y la mayoría tienen regiones de singularidad dentro de su espacio de trabajo (Craig, 2006).

\section{Metodología}

La metodología utilizada en el presente artículo es de desarrollo, simulación y análisis del comportamiento previo de funcionamiento del Robot IRB 2400, mismo que físicamente no se dispone y solo se hace referencia para la aplicación de la simulación, ya que los resultados obtenidos contribuirán a predecir consideraciones a tomar antes de su puesta en marcha. El diagrama en bloques mostrado en la Figura 4, evidencia el algoritmo utilizado en el proceso de desarrollo de la interfaz gráfica de usuario, para lo cual se ha hecho uso del software Matlab y de las ecuaciones matemáticas que modelan, tanto la cinemática directa, como la cinemática diferencial descrita en la sección anterior. Para demostrar su funcionamiento se utilizarán las características geométricas del Robot IRB 2400.

\section{Figura 4.}

Diagrama de bloques para el desarrollo de la interfaz gráfica de usuario.

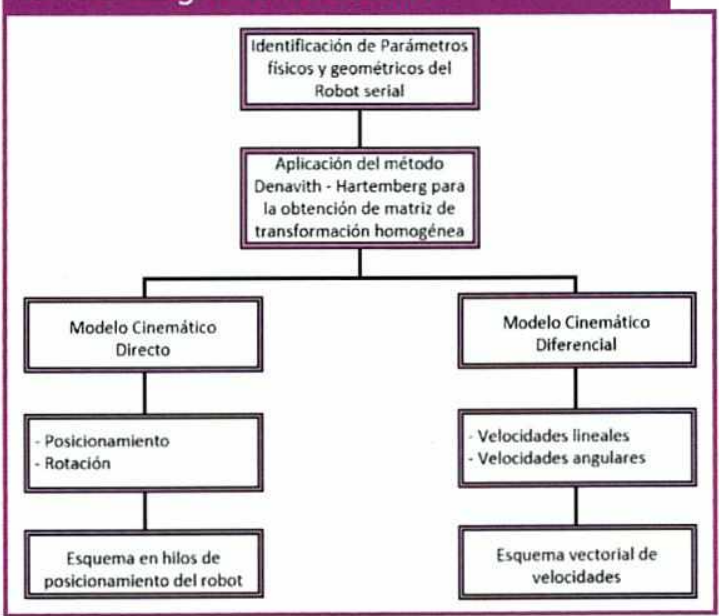

Fuente: Elaboración propia.

Los resultados mostrados en los bloques de posicionamiento, rotación, velocidades lineales y angulares así como los esquemas en hilos de posicionamiento y vectoriales de velocidades, servirán para analizar el comportamiento cinemático previo del robot, con base en la información de entrada de parámetros físicos y geométricos iniciales de robots manipuladores seriales entre dos y seis grados de libertad.

\section{Análisis de Pruebas de Simulación del Robot IRB 2400}

La interfaz desarrollada está destinada exclusivamente para el análisis cinemático de robots manipuladores seriales, desde 2 hasta 6 grados de libertad. La Figura 5, expone su presentación al usuario. En nuestro caso se trata de un robot antropomórfico de 6 grados de libertad.

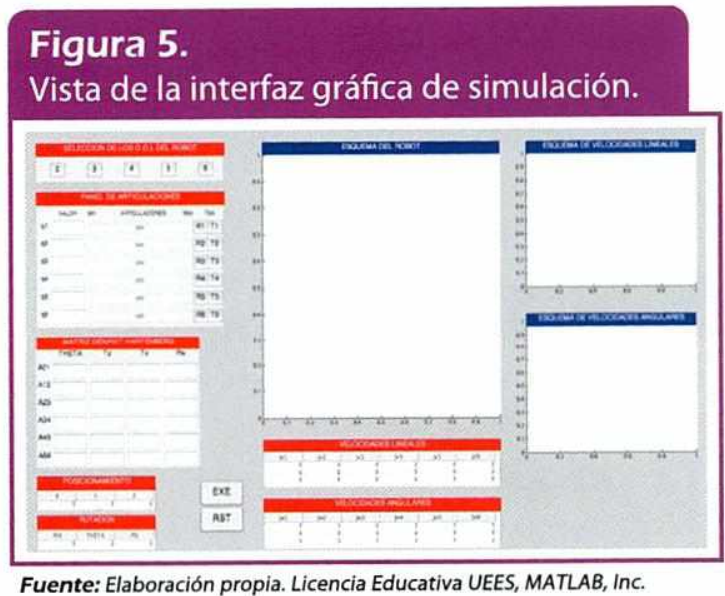


Para poner en marcha la interfaz, se considera las posiciones mostradas en la Figura 2, su estado inicial:( $\mathrm{q} 1=0 ; \mathrm{q} 2=0$; $\mathrm{q} 3=0 ; \mathrm{q} 4=0 ; \mathrm{q} 5=0 ; \mathrm{q} 6=0$ ) y seleccionando los grados de libertad, indicando si el movimiento de las articulaciones obedece al tipo traslacional o rotacional, esto se muestra en la Figura 6. El IRB 2400 al tener una configuración antropomórfica todos los movimientos de sus articulaciones es rotacional. Aunque el valor de q2 muestre un ángulo de $90^{\circ}$, este resulta de la aplicación del método DenavithHartenberg, de allí que el verdadero valor inicial de esta articulación es cero. Según Kin-Huat (2007), la posición cero del manipulador es la posición en la que todas las variables de sus articulaciones son cero. Este procedimiento puede ser útil para comprobar si las posiciones cero del modelo construido son los mismos que los utilizados por el controlador, evitando la necesidad de introducir desviaciones constantes.

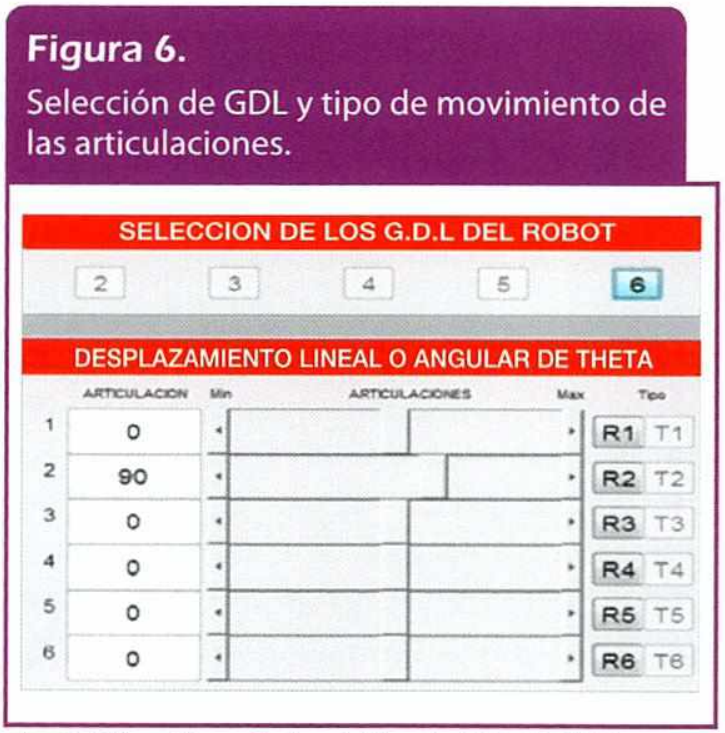

Fuente: Elaboración propia. Licencia Educativa UEES, MATLAB, Inc.

Además, ingresando los parámetros Denavith - Hartemberg se procede a ejecutar la aplicación obteniendo el esquema en hilos, Figura 7, que representa el robot en su estado inicial.

\section{Figura 7.}

Paramétros Denavith - Hartemberg y su

representación esquemática en hilos.

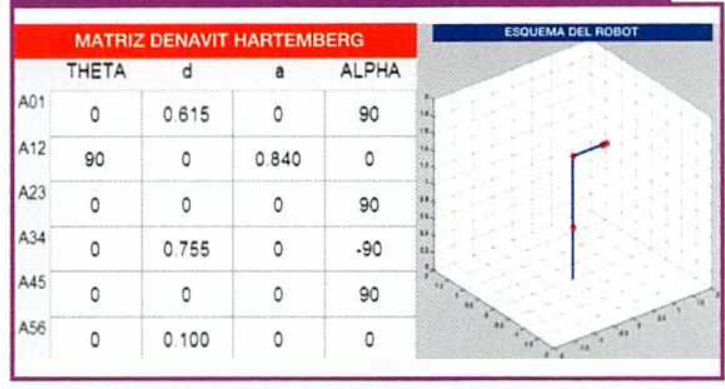

Fuente: Elaboración propia. Licencia Educativa UEES, MATLAB, Inc.

Las coordenadas de posicionamiento tridimensional en metros y ángulos de orientación en grados del efector final, se evidencian en los cuadros que muestra la Figura 8, aquí claramente se demuestra la posición final del elemento de sujeción para la pinza de agarre, también conocida como efector final, evidenciando la geometría de los eslabones definidos por las longitudes $\mathrm{L}_{3}$ y $\mathrm{L}_{4}$ extendidos horizontalmente, los ángulos $(\varnothing, \theta, \psi)$ indican su orientación respecto al sistema de referencia inicial $\left(x_{o}, y_{o}, z_{o}\right)$ mostrado en la Figura 3.

\section{Figura 8.}

Coordenadas de posicionamiento y ángulos del efector final.

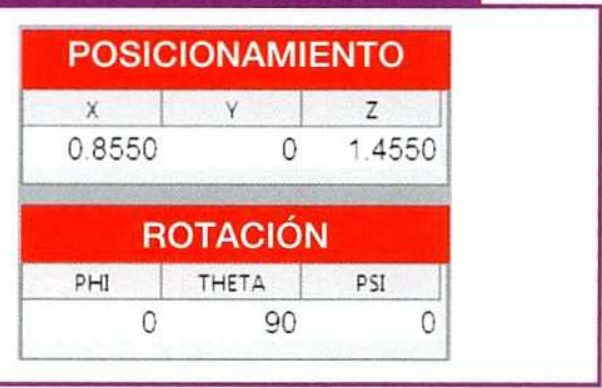

Fuente: Elaboración propia. Licencia Educativa UEES, MATLAB, Inc.

En la Figura 9, la interfaz muestra la matriz Jacobiana geométrica, la misma que está dividida en sus tres primeras filas que representan las velocidades lineales y las tres últimas filas que figuran sus velocidades angulares, evidenciando la relación existente entre velocidades lineales y angulares de sus articulaciones realizada por la cadena cinemática serial, que forma parte del robot en su estructura. 


\section{Figura 9.}

Matriz Jacobiana Geométrica.

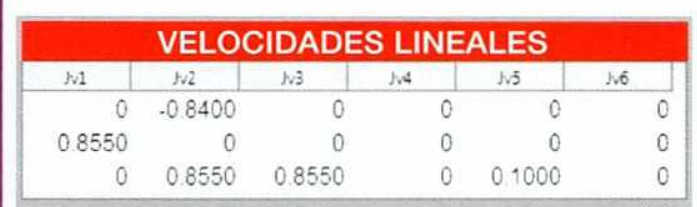

VELOCIDADES ANGULARES

\begin{tabular}{|c|r|r|r|r|r|}
\hline$I_{W 1}$ & $J_{w 2}$ & $J_{w 3}$ & $J_{w 4}$ & $J_{w 5}$ & $J_{w 6}$ \\
\hline 0 & 0 & 0 & 1 & 0 & 1 \\
0 & -1 & -1 & 0 & -1 & 0 \\
1 & 0 & 0 & 0 & 0 & 0 \\
\hline
\end{tabular}

Fuente: Elaboración propia. Licencia Educativa UEES, MATLAB, Inc.
La Figura 10, es la interpretación gráfica de la matriz Jacobiana Geométrica de $6 \times 6$, donde el número de filas representan los seis grados de libertad que el robot posee, el número de columnas y sus seis articulaciones rotacionales. Además, las tres primeras filas de la matriz $J$, corresponden a los vectores de velocidades lineales $J v 1, J v 2, J v 3, J v 4, J v 5$, Jv6, y las 3 últimas filas corresponden a los vectores de velocidades angulares $J w 1, J w 2, J w 3, J w 4$, Jw5, Jw6.

\section{Figura 10.}

Esquema vectorial de velocidades lineales y angulares.

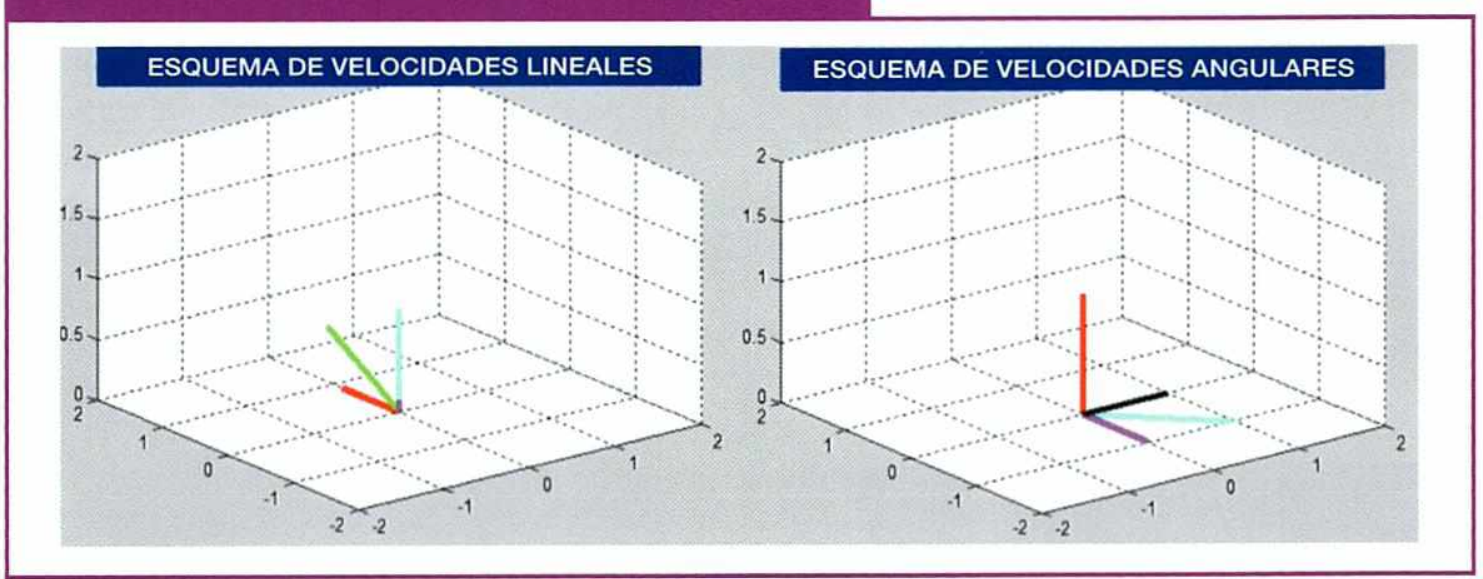

Fuente: Elaboración propia. Licencia Educativa UEES, MATLAB, Inc.

En la Figura 10 se muestra el comportamiento de los vectores de velocidades en sus correspondientes esquemas vectoriales. En el esquema de velocidades lineales se observa que solo existen cuatro vectores, correspondiendo seis, lo que es correcto ya que la inexistencia de dos de ellos $J_{v 4}$ y $J_{v 6}$ significa que en dos de sus articulaciones no se efectuó giro alguno. Además, el hecho que se hayan perdido dos de sus articulaciones, evidencia la presencia de singularidades dentro de su espacio de trabajo, producto a la alineación de los ejes rotacionales en la misma dirección de aquellas articulaciones. La existencia de vectores linealmente dependientes como en las columnas $J v 3$ y $J v 5$ indica que es posible la rotación simultánea de los sistemas de referencia $S_{3}$ y $S_{5}$, esto se evidencia en el esquema como dos vectores paralelos en la misma dirección.

Considerando el esquema de velocidades angulares, la existencia de columnas linealmente dependientes $J w 2, J w 3$ y $J w 5$, indican la existencia de singularidades dentro de su espacio de trabajo, mientras que $J w 4$ y $J w 6$ indican la presencia de singularidades en sus limitaciones geométricas, o fuera de su espacio de trabajo, lo que es consecuencia directa de que en la dirección vertical como en la horizontal, el robot no puede extenderse más allá de sus máximas dimensiones longitudinales. De otro modo, dada la independencia lineal de sus vectores el robot podrá tener cualquier dirección al variar las velocidades angulares de sus articulaciones. El esquema de 
velocidades angulares muestra la presencia de cuatro vectores producto de que dos de sus articulaciones no efectúan giro alguno.

Aunque si bien es cierto, se ha realizado un análisis del robot IRB 2400 consideran- do una configuración inicial $(q 1=0 ; q 2=0$; $\left.q^{3}=0 ; q^{4}=0 ; q^{5}=0 ; q^{6}=0\right)$, se pueden realizar otras pruebas dando estímulos a las articulaciones, por ejemplo considerando: $(q 1=0$; $\left.q 2=0 ; q^{3}=90 ; q^{4}=90 ; q^{5}=0 ; q 6=90\right)$. Los resultados son mostrados en la Figura 11.

\section{Figura 11.}

Configuración $\left(q_{1}=0 ; q_{2}=0 ; q_{3}=90 ; q_{4}=90 ; q_{5}=0 ; q_{6}=90\right)$, resultado de la simulación.

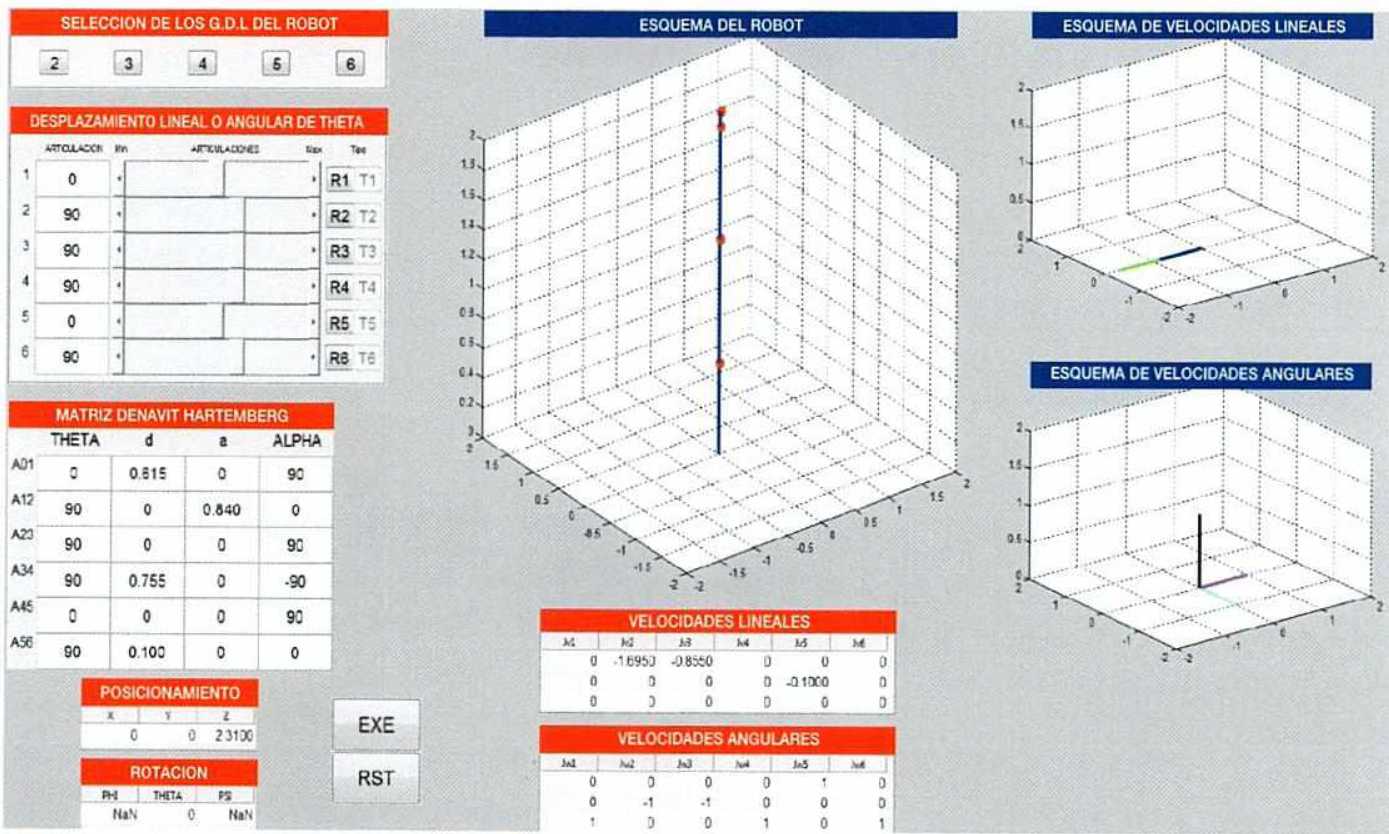

Fuente: Elaboración propia. Licencia Educativa UEES, MATLAB, Inc.

La Figura 11, muestra el despliegue total de la interfaz gráfica de usuario desarrollada, donde se observan los resultados de la matriz Denavith-Hartenberg en caso de lograr rotar 90 grados a las articulaciones q3, q4 y q6; así como también muestra los resultados de la matriz jacobiana y se evidencian los vectores de velocidades angulares y lineales que tienden al paralelismo, interpretándose como una singularidad externa, ya que el robot no podrá ser nunca mayor a sus dimensiones físicas de fabricación. Además, se evidencia el esquema del robot en su posición geométrica final, dada la configuración, esto lo corroboran los indicadores de posicionamiento y orientación.
Sin embargo, la interfaz no solo se limita a realizar pruebas de simulación con el robot IRB 2400, ya que puede simular otros tipos de robots seriales entre 2 y 6 grados de libertad, inclusive si sus articulaciones son solo cartesianas, o solo de revolución sino que además pueden ser combinadas. Una situación a tomar en cuenta al observar la gráfica de las velocidades angulares y lineales, es dada la independencia lineal de los vectores que conforman la matriz Jacobiana, el robot podrá tener cualquier dirección variando las velocidades de sus articulaciones, en este caso $q^{3}, q^{4}$ y $q 6$. Para finalizar, si queremos realizar la prueba con otro tipo de robot serial, pues presionar el botón de RST e 
ingresar nuevos valores.

\section{Conclusiones}

Con base en las ecuaciones del modelo cinemático directo y diferencial se ha desarrollado una interfaz gráfica de simulación, permitiendo conocer cuál es la posición y orientación que adopta el extremo del robot cuando cada una de las variables que fijan la posición u orientación de sus articulaciones toma valores determinados. Además, se ha comprobado su funcionamiento con las dimensiones del manipulador industrial IRB 2400. Se ha realizado la simulación de su posición original y se ha podido determinar las posiciones de su efecto final, así como también su orientación. Además, se ha realizado un análisis de singularidades existentes con base en los resultados proporcionados por su Jacobiana geométrica.

La interfaz gráfica de simulación se la desarrolló para la simulación de manipuladores seriales entre dos y seis grados de libertad, con la opción de seleccionar el tipo de movimiento de sus articulaciones entre rotacionales y prismáticas. Las gráficas esquemáticas en hilos y los diagramas vectoriales de velocidades ayudan a la comprensión analítica del modelo cinemático del robot que se esté tratando.

La utilidad práctica de este tipo de simuladores es conocer previamente el posicionamiento final del efector final y las limitaciones existentes en la estructura física de robots manipuladores industriales, antes de experimentar con uno real.

Sin embargo, un análisis completo del comportamiento del robot implicaría simular también su modelo dinámico, ya que trabajar con el modelo cinemático sería limitado a movimientos suaves del robot; por tanto se recomienda como trabajo futuro incorporar la dinámica del robot, con el fin de analizar su comportamiento a rápidas velocidades y con variaciones de carga en su efector final.

\section{Referencias bibliográficas}

ABB Robotics. (5 de octubre del 2015). ABB. Obtenido de ABB: http://new.abb.com/ products/robotics/es/robots-industriales/ irb-2400

Asada, H., \& Youcef-Toumi, K. (1987). Direct Drive Robots. Cambridge: Press, MIT.

Barrientos, A., Peñín, L., Balaguer, C., \& Aracil, R. (2007). Fundamentos de Robótica. Madrid: Mc Graw Hill.

Bravo, P., \& Reyes, F. (2009). Diseño de un simulador 3D para Robots Manipuladores de 3 grados de libertad. $8^{\circ}$ Congreso Nacional de Mecatrónica, 44-49.

Canudas C., Siciliano B., Bastin G. (1996). Theory of robot control. London: Springer Verlag.

Caraguay, W. (2013). Modelado, identificación $y$ control con base en el modelo dinámico de un robot de dos grados de libertad de accionamiento directo. Universidad Politécnica de Madrid. Obtenido del url: http://repositorio.educacionsuperior.gob. ec/handle/28000/1572

Craig, J. (2006). Robótica. México: Pearson/ Prentice Hall.

Denavit, J., \& Hartenberg, R. (1955). A kinematic notation for lower-pair mechanisms based on matrices. ASME Journal of Applied Mechanics, 215 - 221.

Ertugrul, M., \& Kaynak, O. (1997). Neural network adaptive sliding mode control and its application to SCARA type robot manipulator. International Conference on Robotics and Automation, 2932 - 2937.

Fu, K., González, R., \& Lee, C. (1988). Robótica: Control, detección, visión e inteligencia. Madrid: McGraw-Hill.

Hegde, G. (2008). A Textbook on Industrial Robotics. New Delhi: University Science Press. 
Hoyo, A., Reyes, O., Rebolledo, A., \& Espinoza, L. (2009). Simulación de robots con Matlab y Simulink en escenarios virtuales 3D. I Congreso Iberoamericano de Enseñanza de la Ingeniería, 1-7

Kelly, R., Santibañez, V., Loría, A. (2005). Control of Robot Manipulators in Joint Space. Londres: Springer-Verlag.

Kin-Huat, L., (2009). Industrial Robotics: Programming, Simulation and Applications. Mammendorf: Pro Literatur Verlag.

Kurfess, T. (2005). Robotics and Automation Handbook. New York: CRC Press LLC.

Lewis F., Abdallah C., Dawson D. (1993). Control of robot manipulators. New York: Macmillan Publishers.

Lung-Wen, T. (1999). Robot Analysis. The Mechanics of Serial and Parallel Manipulators. New York: John Willey \& Sons.

Martínez, D., \& Salazar, G. (2007). Diseño y desarrollo de un simulador de robots manipuladores. 6to. Congreso Nacional de Mecatrónica, 104 - 109.

Morales, H., \& Graciano Cruz, M. (2002). EDISIM: Editor y simulador de robots manipuladores. II Congreso Nacional de Electrónica, 1 - 5 .

Muñoz, E., Mosquera, V., \& Gaviria, C. (2011). Evaluación del desempeño de diversos controladores avanzados aplicados a un robot manipulador tipo SCARA. Revista Científica Ingeniería y Desarrollo, 202 -223.

Ollero, A. (2007). Robótica. Manipuladores y Robots Móviles. Barcelona: Alfaomega/ Marcombo.

Paul, R. (1981). Robot Manipulators: Mathematics, Programming, and Control. Massachusetts: The MIT Press.

Reyes, F. (2011). Robótica. Control de Robots Manipuladores. México: Alfaomega/Marcombo.
Reyes, F. (2012). MATLAB Aplicado a robótica y Mecatrónica. México: Alfaomega.

Samson C., Le Borgne M., \& Espinau B. (1991) Robot controls. Oxford: Oxford University Press.

Siciliano, B., Sciavicco, L., Villani, L., \& Oriolo, G. (2009). Robotics. Modelling, Planning and Control. Londres: SpringerVerlag.

Spong, M., \& Vidyasagar, M. (1989). Robot Dynamics and Control. New York: John Willey \& Sons.

Spong, M., Hutchinson, S., \& Vidyasagar, M. (2006). Robot Modeling and Control. New York: John Willey \& Sons.

Vivas, O. (2006). Predictive controls of a SCARA Robot. Revista Chilena de Ingeniería, 135 - 145 .

Yoshikawa, T. (1990). Foundations of Robotics. Massachusetts: MIT Press.

Washington Caraguay Ambuludi

Ingeniero en Electrónica y Telecomunicaciones. Máster en Automática y Robótica.

Docente tiempo completo de la Universidad

Espiritu Santo - Ecuador.

E-mail:wcaraguay@uees.edu.ec

\section{Luis Benavides Castillo}

Ingeniero en Electrónica.

Máster en Administración de Empresas.

Docente tiempo completo de la Universidad

Espiritu Santo - Ecuador.

E-mail: lebenavides@uees.edu.ec 


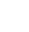

